\title{
SOME NEW RESULTS FOR THE LAGRANGE POLYNOMIALS IN SEVERAL VARIABLES
}

\author{
KUNG-YU CHEN' ${ }^{1}$, SHUOH-JUNG LIU ${ }^{1}$ and H. M. SRIVASTAVA ${ }^{\varpi 2}$
}

(Received 1 March, 2007; revised 29 October, 2007)

\begin{abstract}
In some recent investigations involving certain differential operators for a general family of Lagrange polynomials, Chan et al. encountered and proved a certain summation identity for the Lagrange polynomials in several variables. In the present paper, we derive some generalizations of this summation identity for the Chan-Chyan-Srivastava polynomials in several variables. We also discuss a number of interesting corollaries and consequences of our main results.

2000 Mathematics subject classification: primary 33C05, 33C45: secondary $11 \mathrm{~B} 73$.

Keywords and phrases: Lagrange polynomials, summation identity, Chan-ChyanSrivastava polynomials, differential operators, generating functions, Jacobi polynomials, Stirling numbers of the second kind, Cauchy integral formula, Laguerre polynomials.
\end{abstract}

\section{Introduction}

The familiar (two-variable) polynomials $g_{n}^{(\alpha, \beta)}(x, y)$ generated by

$$
\begin{gathered}
(1-x t)^{-\alpha}(1-y t)^{-\beta}=\sum_{n=0}^{\infty} g_{n}^{(\alpha, \beta)}(x, y) t^{n} \\
\left(|t|<\min \left\{|x|^{-1},|y|^{-1}\right\}\right)
\end{gathered}
$$

are known as the Lagrange polynomials which occur in certain problems in statistics (see, for example, Erdélyi et al. [3, Page 267]; see also Srivastava and Manocha [7, Pages 441-442]). In fact, in terms of the classical Jacobi polynomials $P_{n}^{(\alpha, \beta)}(x)$

\footnotetext{
'Department of Mathematics, Tamkang University, Tamsui 25137, Taiwan, Republic of China; email: kychen@mail.tku.edu.tw, 113014@mail.tku.edu.tw.

${ }^{2}$ Department of Mathematics and Statistics, University of Victoria, Victoria, British Columbia V8W 3P4, Canada, email: harimsri@math.uvic.ca.

(C) Australian Mathematical Society 2007, Serial-fee code 1446-1811/07
} 
defined by (see, for example, Szegö [8, Chapter 4])

$$
\begin{aligned}
P_{n}^{(\alpha, \beta)}(x) & =\sum_{k=0}^{n}\left(\begin{array}{c}
n+\alpha \\
k
\end{array}\right)\left(\begin{array}{c}
n+\beta \\
n-k
\end{array}\right)\left(\frac{x+1}{2}\right)^{k}\left(\frac{x-1}{2}\right)^{n-k} \\
& =\left(\begin{array}{c}
n+\alpha \\
n
\end{array}\right){ }_{2} F_{1}\left(-n, \alpha+\beta+n+1 ; \alpha+1 ; \frac{1-x}{2}\right),
\end{aligned}
$$

where ${ }_{2} F_{1}$ denotes the classical hypergeometric function, it is known that [7, Page 442, Equation 8.5(17)]

$$
g_{n}^{(\alpha, \beta)}(x, y)=(y-x)^{n} P_{n}^{(-\alpha-n,-\beta-n)}\left(\frac{x+y}{x-y}\right) .
$$

The relationship (1.3) can be used in order to deduce numerous properties and characteristics of the (two-variable) Lagrange polynomials from those of the classical Jacobi polynomials.

Recently, a multivariable extension of the Lagrange polynomials in (1.1), generated by

$$
\prod_{j=1}^{r}\left\{\left(1-x_{j} z\right)^{-\alpha_{j}}\right\}=\sum_{k=0}^{\infty} g_{k}^{(\alpha)}(\mathbf{x}) z^{k} \quad\left(|z|<\min \left\{\left|x_{1}\right|^{-1}, \cdots,\left|x_{r}\right|^{-1}\right\}\right),
$$

was introduced and investigated systematically by Chan et al. [1]. Here and subsequently we employ the abbreviations $\alpha$ and $\mathbf{x}$ for $\alpha_{1}+\cdots+\alpha_{r}$ and $x_{1}+\cdots+x_{r}$, respectively. Later we introduce also $\mathrm{n}$ for $n_{1}+\cdots+n_{r}$.

See also several further developments relating to the Chan-Chyan-Srivastava polynomials by (for example) Altın et al. [4] and Erkuş et al. ([5] and [6]).

For the Chan-Chyan-Srivastava polynomials in (1.4), the following explicit representation holds true [1, Page 140, Equation (6)]:

$$
g_{n}^{(\alpha)}(\mathbf{x})=\sum_{k_{1}+\cdots+k_{r}=n}\left(\alpha_{1}\right)_{k_{1}} \cdots\left(\alpha_{r}\right)_{k_{r}} \frac{x_{1}^{k_{1}}}{k_{1} !} \cdots \frac{x_{r}^{k_{r}}}{k_{r} !}
$$

where

$$
(\lambda)_{k}:=\frac{\Gamma(\lambda+k)}{\Gamma(\lambda)}
$$

denotes the Pochhammer symbol (or the shifted factorial). It is known also that [1, Page 146, Equation (34)]

$$
\begin{aligned}
& \sum_{k=0}^{\infty} k^{n} g_{k}^{(\alpha)}(\mathbf{x}) z^{k} \\
& \quad=\prod_{j=1}^{r}\left\{\left(1-x_{j} z\right)^{-\alpha_{j}}\right\} \sum_{k=0}^{n} k ! \cdot S(n, k) \cdot g_{k}^{(\alpha)}\left(\frac{x_{1}}{1-x_{1} z}, \cdots, \frac{x_{r}}{1-x_{r} z}\right) z^{k} \\
& \quad\left(|z|<\min \left\{\left|x_{1}\right|^{-1}, \cdots,\left|x_{r}\right|^{-1}\right\} ; n \in \mathbb{N}_{0}:=\mathbb{N} \cup\{0\} ; \mathbb{N}:=\{1,2,3, \cdots\}\right),
\end{aligned}
$$


which, for $n=0$, corresponds obviously to the defining equation (1.4). Here, and in what follows, $S(n, k)$ denotes the Stirling numbers of the second kind, defined by

so that

$$
S(n, k)=\frac{1}{k !} \sum_{j=0}^{k}(-1)^{k-j}\left(\begin{array}{l}
k \\
j
\end{array}\right) j^{n},
$$

$$
S(n, 0)=\delta_{n, 0}= \begin{cases}1 & (n=0) \\ 0 & (n \in \mathbb{N})\end{cases}
$$

and

$$
\sum_{k=0}^{n}(-1)^{k}(x)_{k} S(n, k)=(-x)^{n},
$$

$\delta_{m, n}$ being the Kronecker symbol.

The main object of this sequel to the aforementioned investigations is to derive a number of generalizations of the summation identities for a family of Jacobi and related hypergeometric polynomials, which were proven recently by (for example) Chen and Srivastava [2]. We also present several interesting corollaries and consequences of our main results.

\section{A set of main results}

We begin by stating and proving a number of useful identities involving the ChanChyan-Srivastava polynomials in (1.4).

LEMMA 2.1. The following generating function holds true for $n \in \mathbb{N}_{0}$ :

$$
\begin{aligned}
\sum_{k=0}^{\infty}[k & +n]_{m} g_{k}^{(\alpha)}(\mathbf{x}) z^{k} \\
& =m ! \prod_{j=1}^{r}\left\{\left(1-x_{j} z\right)^{-\alpha_{j}}\right\} \cdot g_{m}^{\left(\alpha_{i}-n\right)}\left(\frac{x_{1} z}{1-x_{1} z}, \cdots, \frac{x_{r} z}{1-x_{r} z},-1\right),
\end{aligned}
$$

where

$$
[k]_{m}:=k(k-1) \cdots(k-m+1) \quad(m \in \mathbb{N}) \text { and }[k]_{0}=1 .
$$

PROOF. We introduce the closed contours

$$
\mathscr{C}_{\varepsilon} \text { and } \mathscr{C}_{\varepsilon}^{*} \quad\left(0<\varepsilon<\min \left\{\left|x_{1}\right|^{-1}, \ldots,\left|x_{r}\right|^{-1}\right\}\right)
$$


in the complex $\zeta$-plane. These are circles of radius $\varepsilon$ (centred at $\zeta=z$ and $\zeta=0$ respectively), described in the positive (counterclockwise) direction. By Cauchy's integral formula for the derivatives $D_{z}:=d / d z$, we have

$$
\begin{aligned}
& D_{z}^{m}\left\{z^{n} \prod_{j=1}^{r}\left\{\left(1-x_{j} z\right)^{-\alpha_{j}}\right\}\right\} \\
& \quad=\frac{m !}{2 \pi i} \oint_{\mathscr{C}_{i}} \frac{\zeta^{n}}{(\zeta-z)^{m+1}} \prod_{j=1}^{r}\left\{\left(1-x_{j} \zeta\right)^{-\alpha_{j}}\right\} d \zeta \\
& \quad=\frac{m !}{2 \pi i} \oint_{\mathscr{C}_{i}} \frac{(\zeta+z)^{n}}{\zeta^{m+1}} \prod_{j=1}^{r}\left\{\left(1-x_{j}(\zeta+z)\right)^{-\alpha_{j}}\right\} d \zeta \\
& \quad=\frac{m !}{2 \pi i} z^{n} \prod_{j=1}^{r}\left\{\left(1-x_{j} z\right)^{-\alpha_{j}}\right\} \oint_{\mathscr{C}_{i}} \frac{\left(1+\frac{\zeta}{z}\right)^{n}}{\zeta^{m+1}} \prod_{j=1}^{r}\left\{\left(1-\frac{x_{j}}{1-x_{j} z} \zeta\right)^{-\alpha_{j}}\right\} d \zeta \\
& \quad=m ! \cdot z^{n} \prod_{j=1}^{r}\left\{\left(1-x_{j} z\right)^{-\alpha_{j}}\right\} g_{m}^{\left(\alpha_{1}, \cdots, \alpha_{r},-n\right)}\left(\frac{x_{1}}{1-x_{1} z}, \cdots, \frac{x_{r}}{1-x_{r} z},-\frac{1}{z}\right) \\
& \quad=m ! \cdot z^{n-m} \prod_{j=1}^{r}\left\{\left(1-x_{j} z\right)^{-\alpha_{j}}\right\} g_{m}^{(\alpha,-n)}\left(\frac{x_{1} z}{1-x_{1} z}, \cdots, \frac{x_{r} z}{1-x_{r} z},-1\right) .
\end{aligned}
$$

The definition (1.4) can be used to observe further that

$$
\begin{aligned}
D_{z}^{m}\left\{z^{n} \prod_{j=1}^{r}\left\{\left(1-x_{j} z\right)^{-\alpha_{j}}\right\}\right\} & =D_{z}^{m}\left\{z^{n} \sum_{k=0}^{\infty} g_{k}^{(\alpha)}(\mathbf{x}) z^{k}\right\} \\
& =z^{n-m} \sum_{k=0}^{\infty}[k+n]_{m} g_{k}^{(\alpha)}(\mathbf{x}) z^{k}
\end{aligned}
$$

which, in association with (2.2), yields the generating function (2.1) asserted by Lemma 2.1 .

REMARK 1. By setting $n=m$ and $n=0$ in the assertion (2.1) of Lemma 2.1, we obtain the following simpler generating functions:

$\sum_{k=0}^{\infty}(k+1)_{m} g_{k}^{(\alpha)}(\mathbf{x}) z^{k}=m ! \prod_{j=1}^{r}\left\{\left(1-x_{j} z\right)^{-\alpha_{j}}\right\} g_{m}^{(\alpha,-m)}\left(\frac{x_{1} z}{1-x_{1} z}, \cdots, \frac{x_{r} z}{1-x_{r} z},-1\right)$

and

$\sum_{k=0}^{\infty}(k+1)_{m} g_{k+m}^{(\alpha)}(\mathbf{x}) z^{k}=m ! \prod_{j=1}^{r}\left\{\left(1-x_{j} z\right)^{-\alpha_{j}}\right\} g_{m}^{(\alpha)}\left(\frac{x_{1}}{1-x_{1} z}, \cdots, \frac{x_{r}}{1-x_{r} z}\right)$,

respectively. 
LEMMA 2.2. The following summation identity holds true:

$$
\begin{aligned}
& \sum_{k=0}^{p}[k+n]_{m} g_{k}^{(\alpha)}(\mathbf{x}) g_{p-k}^{(-\alpha-\mathrm{n})}(\mathbf{x}) \\
& \quad=\delta_{p, n_{1}+\cdots+n_{r}} \prod_{j=1}^{r}\left\{\left(-x_{j}\right)^{n_{j}}\right\}(-1)^{m}\left(\alpha_{1}+\cdots+\alpha_{r}-n\right)_{m}+\psi_{1}(\boldsymbol{\alpha})
\end{aligned}
$$

$\left(m, p, n, n_{1}, \cdots, n_{r} \in \mathbb{N}_{0} ; n_{j} \geq m-1 \quad(j=1, \cdots, r) ; p \geq n_{1}+\cdots+n_{r}\right)$.

Here and subsequently

$$
\psi_{k}(\alpha)=\sum_{s=k}^{r}\left[\delta_{n_{s}, m-1}\left(\alpha_{s}\right)_{m} x_{s}^{p} \prod_{k \leq j \leq r(j \neq s)}\left\{\left(1-\frac{x_{j}}{x_{s}}\right)^{n_{j}}\right\}\right] \quad(k=1,2) .
$$

PROOF. First of all, by applying the generating functions (1.4) and (2.1), we have

$$
\begin{aligned}
\sum_{p=0}^{\infty} Q_{p}(\mathbf{x}) z^{p} & =\left(\sum_{k=0}^{\infty}[k+n]_{m} g_{k}^{(\alpha)}(\mathbf{x}) z^{k}\right)\left(\sum_{k=0}^{\infty} g_{k}^{(-\alpha-n)}(\mathbf{x}) z^{k}\right) \\
& =m ! \prod_{j=1}^{r}\left\{\left(1-x_{j} z\right)^{n_{j}}\right\} g_{m}^{(\alpha-n)}\left(\frac{x_{1} z}{1-x_{1} z}, \cdots, \frac{x_{r} z}{1-x_{r} z},-1\right)
\end{aligned}
$$

where, for convenience,

$$
Q_{p}(\mathbf{x}):=\sum_{k=0}^{p}[k+n]_{m} g_{k}^{(\alpha)}(\mathbf{x}) \cdot g_{p-k}^{(-\alpha-n)}(\mathbf{x})
$$

Thus, in view of the Cauchy integral formula again, we have

$$
Q_{p}(\mathbf{x})=\frac{m !}{2 \pi i} \oint_{\mathscr{C}_{i}} \frac{1}{z^{p+1}} \prod_{j=1}^{r}\left\{\left(1-x_{j} z\right)^{n_{j}}\right\} g_{m}^{(\alpha,-n)}\left(\frac{x_{1} z}{1-x_{1} z}, \cdots, \frac{x_{r} z}{1-x_{r} z},-1\right) d z
$$

By a change of variable given by

$$
z=\frac{1}{\zeta} \text { and } \quad d z=-\frac{1}{\zeta^{2}} d \zeta
$$

(2.5) readily yields

$$
\begin{aligned}
Q_{p}(\mathbf{x})= & m ! \sum_{k_{1}+\cdots+k_{r}+s=m}\left(\alpha_{1}\right)_{k_{1}} \cdots\left(\alpha_{r}\right)_{k_{r}}(-n)_{s} \frac{x_{1}^{k_{1}}}{k_{1} !} \cdots \frac{x_{r}^{k_{r}}}{k_{r} !} \frac{(-1)^{s}}{s !} \\
& \cdot \frac{1}{2 \pi i} \oint_{\mathscr{C}_{i / k}^{*}} \frac{\zeta^{p-n_{1}-\cdots-n_{r}} \prod_{j=1}^{r}\left\{\left(\zeta-x_{j}\right)^{n_{j}-k_{j}+1}\right\}}{\zeta \prod_{j=1}^{r}\left\{\left(\zeta-x_{j}\right)\right\}} d \zeta,
\end{aligned}
$$


where the closed contour $\mathscr{C}_{1 / \varepsilon}^{*}$ in the complex $\zeta$-plane is a circle (centred at $\zeta=0$ ) of radius $1 / \varepsilon$, which is described in the positive (counterclockwise) direction.

Since, by hypothesis,

$$
p \geq n_{1}+\cdots+n_{r} \quad \text { and } \quad n_{j} \geq m-1 \quad(j=1, \ldots, r),
$$

it is easy to see that

$$
\zeta^{p-n_{1}-\cdots-n_{r}} \prod_{j=1}^{r}\left\{\left(\zeta-x_{j}\right)^{n_{j}-k_{j}+1}\right\}
$$

is a polynomial in $\zeta$, and hence it is analytic under these constraints on the nonnegative integers $p, m, r, n_{1}, \cdots, n_{r}$ and $k_{1}, \cdots, k_{r}$. Thus, for convenience, if we put

$$
f(\zeta):=\zeta^{p-n_{1}-\cdots-n_{r}-1} \prod_{j=1}^{r}\left\{\left(\zeta-x_{j}\right)^{n_{j}-k_{j}}\right\},
$$

it follows (by the Cauchy residue theorem) that

$$
\begin{aligned}
Q_{p}(\mathbf{x})= & \delta_{p, n_{1}+\cdots+n_{r}} m ! \sum_{k_{1}+\cdots+k_{r}+s=m}\left(\alpha_{1}\right)_{k_{1}} \cdots\left(\alpha_{r}\right)_{k_{r}}(-n)_{s} \cdot \frac{x_{1}^{k_{1}}}{k_{1} !} \cdots \frac{x_{r}^{k_{r}}}{k_{r} !} \frac{(-1)^{s}}{s !} \\
& \cdot \lim _{\zeta \rightarrow 0}\{(\zeta-0) f(\zeta)\}+\psi_{1}(\alpha) \\
= & \delta_{p, n_{1}+\cdots+n_{r}} m !(-1)^{m} \prod_{j=1}^{r}\left\{\left(-x_{j}\right)^{n_{j}}\right\} g_{m}^{(\alpha,-n)}(1, \cdots, 1,1)+\psi_{1}(\alpha) .
\end{aligned}
$$

It is easily seen from the definition (1.4) that

$$
g_{n}^{(\alpha)}(x, \cdots, x)=\left(\begin{array}{c}
\alpha_{1}+\cdots+\alpha_{r}+n-1 \\
n
\end{array}\right) x^{n}=\frac{\left(\alpha_{1}+\cdots+\alpha_{r}\right)_{n}}{n !} x^{n},
$$

which, upon setting $x=1$, immediately yields

$$
g_{n}^{(\alpha)}(1, \cdots, 1)=\left(\begin{array}{c}
\alpha_{1}+\cdots+\alpha_{r}+n-1 \\
n
\end{array}\right)=\frac{\left(\alpha_{1}+\cdots+\alpha_{r}\right)_{n}}{n !} .
$$

By appropriately combining (2.6) and (2.8), we obtain the summation identity (2.4) asserted by Lemma 2.2 .

Clearly, Lemma 2.2 is equivalent to the following result:

$$
\begin{aligned}
& \sum_{k=0}^{p}[k+n]_{m} g_{k}^{(-\alpha-n)}(\mathbf{x}) g_{p-k}^{(\alpha)}(\mathbf{x}) \\
& \quad=\delta_{p, n_{1}+\cdots+n_{r}} \prod_{j=1}^{r}\left\{\left(-x_{j}\right)^{n_{j}}\right\}(-1)^{m}\left(-\alpha_{1}-\cdots-\alpha_{r}-p-n\right)_{m}+\psi_{1}(-\boldsymbol{\alpha}-\mathbf{n}) \\
& \quad\left(m, p, n, n_{1}, \cdots, n_{r} \in \mathbb{N}_{0} ; n_{j} \geq m-1 \quad(j=1, \cdots, r) ; p \geq n_{1}+\cdots+n_{r}\right) .
\end{aligned}
$$


Next, since (by definition) we have

$$
(-\chi)_{k}=(-1)^{k}[\chi]_{k} \quad\left(k \in \mathbb{N}_{0} ; \chi \in \mathbb{C}\right),
$$

it readily follows from (1.8) that (see also [9, Page 15])

$$
k^{n}=\sum_{j=1}^{n} S(n, j) \cdot[k]_{j}
$$

Furthermore, it is easily seen by setting $m=0$ in (2.9) that

$$
\begin{gathered}
\sum_{k=0}^{p} g_{k}^{(-\alpha-n)}(\mathbf{x}) g_{p-k}^{(\alpha)}(\mathbf{x})=\delta_{p, n_{1}+\cdots+n_{r}} \prod_{j=1}^{r}\left\{\left(-x_{j}\right)^{n_{j}}\right\} \\
\left(p, n_{1}, \cdots, n_{r} \in \mathbb{N}_{0} ; p \geq n_{1}+\cdots+n_{r}\right) .
\end{gathered}
$$

By making use of (1.7), (1.8) and (2.9)-(2.11), our main summation formula involving the multivariable Lagrange polynomials $g_{n}^{(\alpha)}(\mathbf{x})$ is given by Theorem 2.3 below.

THEOREM 2.3. For every polynomial $\mathscr{P}_{m}(x)$ of degree $m$ in $x$,

$$
\begin{aligned}
& \sum_{k=0}^{p} \mathscr{P}_{m}(k) g_{k}^{(-\alpha-n)}(\mathbf{x}) g_{p-k}^{(\alpha)}(\mathbf{x})= \delta_{p, n_{1}+\cdots+n_{r}} \prod_{j=1}^{r}\left\{\left(-x_{j}\right)^{n_{j}}\right\} \mathscr{P}_{m}\left(\alpha_{1}+\cdots+\alpha_{r}+p\right) \\
&+\frac{(-1)^{m}}{m !} \mathscr{P}_{m}^{(m)}(0) \psi_{1}(\alpha) \\
&\left(m, p, n_{1}, \cdots, n_{:} \in \mathbb{N}_{0} ; n_{j} \geq m-1(j=1, \cdots, r) ; p \geq n_{1}+\cdots+n_{r}\right) .
\end{aligned}
$$

PROOF. Upon setting

$$
\mathscr{P}_{m}(k)=\sum_{\ell=0}^{m} c_{\ell} k^{\ell},
$$

we have

$$
\begin{aligned}
& \sum_{k=0}^{p} \mathscr{P}_{m}(k) g_{k}^{(-\alpha-n)}(\mathbf{x}) g_{p-k}^{(\alpha)}(\mathbf{x}) \\
& \quad=\sum_{k=0}^{p}\left[c_{0}+\sum_{\ell=1}^{m} c_{\ell}\left(\sum_{j=1}^{\ell} S(\ell, j)[k]_{j}\right)\right] \cdot g_{k}^{(-\alpha-n)}(\mathbf{x}) g_{p-k}^{(\alpha)}(\mathbf{x}) \\
& \quad=c_{0} \delta_{p, n_{1}+\cdots+n_{r}} \prod_{q=1}^{r}\left\{\left(-x_{q}\right)^{n_{q}}\right\}+\sum_{\ell=1}^{m} \sum_{j=0}^{\ell} c_{\ell} S(\ell, j) \cdot \sum_{k=0}^{p}[k]_{j} g_{k}^{(-\alpha-n)}(\mathbf{x}) g_{p-k}^{(\alpha)}(\mathbf{x}) \\
&=\delta_{p, n_{1}+\cdots+n_{r}} \prod_{j=1}^{r}\left\{\left(-x_{j}\right)^{n_{j}}\right\} \mathscr{P}_{m}\left(\alpha_{1}+\cdots+\alpha_{r}+p\right)+\frac{(-1)^{m}}{m !} \mathscr{P}_{m}^{(m)}(0) \psi_{1}(\alpha),
\end{aligned}
$$

which precisely is the assertion (2.12) of Theorem 2.3 . 
REMARK 2 . Theorem 2.3 can be proven alternatively by applying the generating functions (1.4) and (1.6) appropriately. Furthermore, if we set

$$
n_{j}=m-1 \quad(j=1, \cdots, r)
$$

in Theorem 2.3, we deduce Corollary 2.4 below.

COROLlaRY 2.4. For every polynomial $\mathscr{P}_{m}(x)$ of degree $m$ in $x$,

$$
\begin{aligned}
& \sum_{k=0}^{p} \mathscr{P}_{m}(k) g_{k}^{\left(-\alpha_{1}-m+1, \cdots,-\alpha_{r}-m+1\right)}(\mathbf{x}) g_{p-k}^{(\alpha)}(\mathbf{x}) \\
&= \delta_{p, r(m-1)} \prod_{j=1}^{r}\left\{\left(-x_{j}\right)^{m-1}\right\} \mathscr{P}_{m}\left(\alpha_{1}+\cdots+\alpha_{r}+p\right) \\
&+\frac{(-1)^{m}}{m !} \mathscr{P}_{m}^{(m)}(0) \sum_{s=1}^{r}\left[\left(\alpha_{s}\right)_{m} x_{s}^{p} \prod_{1 \leq j \leq r(j \neq s)}\left\{\left(1-\frac{x_{j}}{x_{s}}\right)^{m-1}\right\}\right] \\
&\left(m \in \mathbb{N} ; p \in \mathbb{N}_{0} ; p \geq r(m-1)\right) .
\end{aligned}
$$

REMARK 3. In view of the relationship (1.3), the assertion (2.12) of Theorem 2.3 (with $r=2$ ) yields the following summation formula for the classical Jacobi polynomials defined by (1.2).

COROLLARY 2.5. For every polynomial $\mathscr{P}_{m}(x)$ of degree $m$ in $x$,

$$
\begin{aligned}
\sum_{k=0}^{p} \mathscr{P}_{m}(k) P_{k}^{\left(\alpha+n_{1}-k, \beta+n_{2}-k\right)}(x) P_{p-k}^{(-\alpha-p+k,-\beta-p+k)}(x) \\
=\delta_{p, n_{1}+n_{2}}\left(\frac{x+1}{2}\right)^{n_{1}}\left(\frac{x-1}{2}\right)^{n_{2}} \mathscr{P}_{m}(\alpha+\beta+p)+\frac{(-1)^{m}}{m !} \mathscr{P}_{m}^{(m)}(0) \\
\cdot\left[\delta_{n_{1}, m-1}(\alpha)_{m}(-1)^{p}\left(\frac{x+1}{2}\right)^{p-n_{2}}+\delta_{n_{2}, m-1}(\beta)_{m}\left(\frac{1-x}{2}\right)^{p-n_{1}}\right] \\
\left(m, p, n_{1}, n_{2} \in \mathbb{N}_{0} ; n_{1}, n_{2} \geq m-1 ; p \geq n_{1}+n_{2}\right) .
\end{aligned}
$$

PROOF. Applying the relationship (1.3) in the form

$$
P_{k}^{(-\alpha-k,-\beta-k)}\left(\frac{x+y}{x-y}\right)=(y-x)^{-k} g_{k}^{(\alpha, \beta)}(x, y),
$$

we find that 


$$
\begin{gathered}
\sum_{k=0}^{p} \mathscr{P}_{m}(k) P_{k}^{\left(\alpha+n_{1}-k, \beta+n_{2}-k\right)}\left(\frac{x+y}{x-y}\right) P_{p-k}^{(-\alpha-p+k,-\beta-p+k)}\left(\frac{x+y}{x-y}\right) \\
=(y-x)^{-p} \sum_{k=0}^{p} \mathscr{P}_{m}(k) g_{k}^{\left(-\alpha-n_{1},-\beta-n_{2}\right)}(x, y) g_{p-k}^{(\alpha, \beta)}(x, y) .
\end{gathered}
$$

By means of (2.12), the right-hand side equals

$$
\begin{aligned}
& (y-x)^{-p}\left\{\delta_{p, n_{1}+n_{2}}(-x)^{n_{1}}(-y)^{n_{2}} \mathscr{P}_{m}(\alpha+\beta+p)\right. \\
& \left.\quad+\frac{(-1)^{m}}{m !} \mathscr{P}_{m}^{(m)}(0)\left[\delta_{n_{1}, m-1}(\alpha)_{m} x^{p}\left(1-\frac{y}{x}\right)^{n_{2}}+\delta_{n_{2}, m-1}(\beta)_{m} y^{p}\left(1-\frac{x}{y}\right)^{n_{1}}\right]\right\} .
\end{aligned}
$$

Finally, the assertion (2.13) of Corollary 2.5 follows immediately upon setting $y=x(x-1) /(x+1)$ in (2.14).

Alternatively, since [7, Page 441, Equation 8.5(16)]

$$
P_{n}^{(\alpha-n, \beta-n)}(x)=g_{n}^{(-\alpha,-\beta)}\left(-\frac{x+1}{2},-\frac{x-1}{2}\right),
$$

it is easily seen that

$$
\begin{aligned}
& \sum_{k=0}^{p} \mathscr{P}_{m}(k) P_{k}^{\left(\alpha+n_{1}-k, \beta+n_{2}-k\right)}(x) P_{p-k}^{(-\alpha-p+k,-\beta-p+k)}(x) \\
& \quad=\sum_{k=0}^{p} \mathscr{P}_{m}(\dot{\kappa}) S_{k}^{\left(-\alpha-n_{1},-\beta-n_{2}\right)}\left(-\frac{x+1}{2},-\frac{x-1}{2}\right) \cdot g_{p-k}^{(\alpha, \beta)}\left(-\frac{x+1}{2},-\frac{x-1}{2}\right),
\end{aligned}
$$

which, in light of (2.12), readily yields the assertion (2.13) of Corollary 2.5.

REMARK 4. It is not difficult to verify that the relationships (1.3) and (2.15) are equivalent. It is known also that [7, Page 452, Problem 25]

$$
g_{n}^{(\alpha, \beta)}(x, y)=y^{n} P_{n}^{(\alpha+\beta-1,-\beta-n)}\left(\frac{2 x-y}{y}\right),
$$

which, upon setting $y=2 x /(x+1)$, yields

$$
P_{n}^{(\alpha+\beta-1,-\beta-n)}(x)=\left(\frac{x+1}{2 x}\right)^{n} g_{n}^{(\alpha, \beta)}\left(x, \frac{2 x}{x+1}\right) .
$$

Thus, by making use of the relationship (2.17), we obtain

$$
\begin{aligned}
\sum_{k=0}^{p} \mathscr{P}_{m}(k) P_{k}^{\left(-\alpha-\beta-n_{1}-n_{2}-1, \beta+n_{2}-k\right)}(x) P_{p-k}^{(\alpha+\beta-1,-\beta-p+k)}(x) \\
\quad=\left(\frac{x+1}{2 x}\right)^{p} \sum_{k=0}^{p} \mathscr{P}_{m}(k) g_{k}^{\left(-\alpha-n_{1},-\beta-n_{2}\right)}\left(x, \frac{2 x}{x+1}\right) g_{p-k}^{(\alpha, \beta)}\left(x, \frac{2 x}{x+1}\right) .
\end{aligned}
$$


Consequently, by applying (2.12), we arrive at the following summation formula analogous to (2.13):

$$
\begin{aligned}
\sum_{k=0}^{p} \mathscr{P}_{m}(k) P_{k}^{\left(-\alpha-\beta-n_{1}-n_{2}-1, \beta+n_{2}-k\right)}(x) P_{p-k}^{(\alpha+\beta-1,-\beta-p+k)}(x) \\
=\delta_{p, n_{1}+n_{2}}(-1)^{p}\left(\frac{x+1}{2}\right)^{n_{1}} \mathscr{P}_{m}(\alpha+\beta+p)+\frac{(-1)^{m}}{m !} \mathscr{P}_{m}^{(m)}(0) \\
\cdot\left[\delta_{n_{1}, m-1}(\alpha)_{m}\left(\frac{x+1}{2}\right)^{p}\left(\frac{x-1}{x+1}\right)^{n_{2}}+\delta_{n_{2}, m-1}(\beta)_{m}\left(\frac{1-x}{2}\right)^{n_{1}}\right] \\
\quad\left(m, p, n_{1}, n_{2} \in \mathbb{N}_{0} ; n_{1}, n_{2} \geq m-1 ; p \geq n_{1}+n_{2}\right) .
\end{aligned}
$$

REMARK 5. By setting

$$
\begin{array}{ll}
\alpha \longmapsto-\alpha-\beta-r, & \beta \longmapsto \beta-n+r+1, \\
n_{1} \longmapsto r-1 & \text { and } \quad n_{2} \longmapsto n-r-1,
\end{array}
$$

and considering the special case when $p \geq n_{1}+n_{2}+1$, (2.18) would yield the following known result for the classical Jacobi polynomials $P_{n}^{(\alpha, \beta)}(x)$ [2, Page 3301, Theorem 3]:

$$
\begin{aligned}
\sum_{k=j}^{l} \mathscr{P}_{m}(k) P_{k-j}^{(\alpha, \beta-k+j)}(x) P_{l-k}^{(-\alpha-n,-\beta+n-r+k-l-1)}(x) \\
=\frac{\mathscr{P}_{m}^{(m)}(0)}{m !}\left[\delta_{n, m+r}(-\beta)_{m}\left(\frac{1-x}{2}\right)^{r-1}\right. \\
\left.\quad+\delta_{r, m}(\alpha+\beta+1)_{m}\left(\frac{x-1}{2}\right)^{n-r-1}\left(\frac{x+1}{2}\right)^{l-j+m-n+1}\right] \\
\quad\left(j, l, m, n, r \in \mathbb{N}_{0} ; l-j+1 \geq n \geq m+r ; r \geq m\right) .
\end{aligned}
$$

REMARK 6. In its special case when $n_{1}=n_{2}=m$, we find from the assertion (2.13) of Corollary 2.5 that

$$
\begin{aligned}
& \sum_{k=0}^{p} \mathscr{P}_{m}(k) P_{k}^{(\alpha+m-k, \beta+m-k)}(x) P_{p-k}^{\left(-\alpha-p+k_{,}-\beta-p+k\right)}(x) \\
& \quad=\delta_{p, 2 m}\left(\frac{x^{2}-1}{4}\right)^{m} \mathscr{P}_{m}(\alpha+\beta+p) \quad\left(m \in \mathbb{N}_{0} ; p \geq 2 m\right),
\end{aligned}
$$

which, upon setting $m=0$, yields the following interesting sum:

$$
\sum_{k=0}^{p} P_{k}^{(\alpha-k, \beta-k)}(x) P_{p-k}^{(-\alpha-p+k,-\beta-p+k)}(x)=0 \quad(p \in \mathbb{N}) .
$$




\section{Further results and consequences}

For a nonnegative integer $n$, the Laguerre polynomials $L_{n}^{(\alpha)}(x)$ of index $\alpha$ and degree $n$ in $x$ are defined by

$$
L_{n}^{(\alpha)}(x)=\sum_{k=0}^{n}\left(\begin{array}{c}
n+\alpha \\
n-k
\end{array}\right) \frac{(-x)^{k}}{k !}=\left(\begin{array}{c}
n+\alpha \\
n
\end{array}\right), F_{1}(-n ; \alpha+1 ; x) .
$$

The Laguerre polynomials $L_{n}^{(\alpha)}(x)$ and the Jacobi polynomials $P_{n}^{(\alpha, \beta)}(x)$ are indeed related by means of the following familiar limit relationship [7, Page 131, Equation 2.5(1)]:

$$
L_{n}^{(\alpha)}(x)=\lim _{|\beta| \rightarrow \infty}\left\{P_{n}^{(\alpha, \beta)}\left(1-\frac{2 x}{\beta}\right)\right\} .
$$

Making use of (3.1), we find from (2.19) with

$$
x \longmapsto 1-\frac{2 x}{\beta} \text { and }|\beta| \longrightarrow \infty
$$

that

$$
\sum_{k=0}^{p} \mathscr{P}_{m}(k) L_{k}^{(\alpha+m-k)}(x) L_{p-k}^{(-\alpha-p+k)}(-x)=\delta_{p, 2 m} \frac{(-x)^{m}}{m !} \mathscr{P}_{m}^{(m)}(0) \quad\left(m \in \mathbb{N}_{0} ; p \geq 2 m\right),
$$

which, upon setting $m=0$, yields the following companion of (2.20):

$$
\sum_{k=0}^{p} L_{k}^{(\alpha-k)}(x) L_{p-k}^{(-\alpha-p+k)}(-x)=0 \quad(p \in \mathbb{N}) .
$$

REMARK 7. By setting

$$
x_{1}=\cdots=x_{r}=x
$$

and using (2.7), (2.12) would yield the following result:

$$
\begin{aligned}
& \sum_{k=0}^{p} \mathscr{P}_{m}(k) \frac{\left(-\alpha_{1}-\cdots-\alpha_{r}-n_{1}-\cdots-n_{r}\right)_{k}\left(\alpha_{1}+\cdots+\alpha_{r}\right)_{p-k}}{k !(p-k) !} x^{p} \\
& \quad=\delta_{p, n_{1}+\cdots+n_{r}}(-x)^{n_{1}+\cdots+n_{r}} \mathscr{P}_{m}\left(\alpha_{1}+\cdots+\alpha_{r}+p\right) \\
& \left(m, p, n_{1}, \cdots, n_{r} \in \mathbb{N}_{0} ; n_{j} \geq m-1 \quad(j=1, \cdots, r) ; p \geq n_{1}+\cdots+n_{r}\right) .
\end{aligned}
$$

Thus, by equating the coefficients of $x^{p}$, we find from (3.2) that

$$
\sum_{k=0}^{p} \mathscr{P}_{m}(k) \frac{(-\alpha-n)_{k}(\alpha)_{p-k}}{k !(p-k) !}=0 \quad\left(m, p, n \in \mathbb{N}_{0} ; p>n \geq m\right)
$$

and 


$$
\sum_{k=0}^{p} \mathscr{P}_{m}(k) \frac{(-\alpha-p)_{k}(\alpha)_{p-k}}{k !(p-k) !}=(-1)^{p} \mathscr{P}_{m}(\alpha+p) \quad\left(m, p \in \mathbb{N}_{0} ; p \geq m\right) .
$$

Furthermore, if we set $\alpha=1$ in (3.3) and (3.4), we get

$$
\sum_{k=0}^{p}(-1)^{k}\left(\begin{array}{c}
n+1 \\
k
\end{array}\right) \mathscr{P}_{m}(k)=0 \quad\left(m, p, n \in \mathbf{N}_{0} ; p>n \geq m\right)
$$

and

$$
\sum_{k=0}^{p}(-1)^{k}\left(\begin{array}{c}
p+1 \\
k
\end{array}\right) \mathscr{P}_{m}(k)=(-1)^{p} \mathscr{P}_{m}(p+1) \quad\left(m, p \in \mathbf{N}_{0} ; p \geq m\right) .
$$

Next, in order to find a summation formula involving two Lagrange polynomials with different indices, one of which is independent of the degree $n$ and the other of which is dependent on the degree $n$, we first derive the following relationship.

LEMMA 3.1. For the two-variable Lagrange polynomials $g_{n}^{(\alpha . \beta)}(x, y)$,

$$
g_{n}^{(\alpha, \beta-n)}(x, y)=g_{n}^{(\alpha,-\alpha-\beta+1)}(x-y,-y) .
$$

PROOF. We have

$$
\begin{aligned}
\sum_{n=0}^{\infty} g_{n}^{(\alpha . \beta-n)}(x, y) z^{n} & =\sum_{n=0}^{\infty} \frac{z^{n}}{2 \pi i} \oint_{\mathscr{C}_{i}^{*}} \frac{(1-x \zeta)^{-\alpha}(1-y \zeta)^{n-\beta}}{\zeta^{n+1}} d \zeta \\
& =\sum_{n=0}^{\infty} \frac{1}{2 \pi i} \oint_{\mathscr{C}_{i}} \frac{(1-x \zeta)^{-\alpha}(1-y \zeta)^{-\beta}}{\zeta}\left(\frac{z(1-y \zeta)}{\zeta}\right)^{n} d \zeta \\
& =\frac{1}{2 \pi i} \oint_{\mathscr{C}_{i}}(1-x \zeta)^{-\alpha}(1-y \zeta)^{-\beta} \frac{1}{1+y z}\left(\zeta-\frac{z}{1+y z}\right)^{-1} d \zeta \\
& =\left(1-\frac{x z}{1+y z}\right)^{-\alpha}\left(1-\frac{y z}{1+y z}\right)^{-\beta} \frac{1}{1+y z} \\
& =[1-(x-y) z]^{-\alpha}(1+y z)^{\alpha+\beta-1} \\
& =\sum_{n=0}^{\infty} g_{n}^{(\alpha,-\alpha-\beta+1)}(x-y,-y) z^{n},
\end{aligned}
$$

where the closed contour $\mathscr{C}_{\varepsilon}^{*} \quad\left(0<\varepsilon<\min \left\{|x|^{-1},|y|^{-1}\right\}\right)$ in the complex $\zeta$-plane is a circle of radius $\varepsilon$ (centred at $\zeta=0$ ), which is described in the positive (counterclockwise) direction and we have tacitly assumed that

$$
|z|<\min \left\{|x|^{-1},|y|^{-1},\left|\frac{\zeta}{1-y \zeta}\right|\right\}
$$

on the contour $\mathscr{C}_{\varepsilon}^{*}$. By equating the coefficients of $z^{n}$ in the preceding generatingfunction relationship, we immediately obtain the assertion (3.5) of Lemma 3.1. 
REMARK 8. Since

$$
P_{n}^{(\alpha, \beta)}(-x)=(-1)^{n} P_{n}^{(\beta, \alpha)}(x)
$$

and

$$
g_{n}^{(\alpha, \beta)}(x, y)=g_{n}^{(\beta, \alpha)}(y, x),
$$

by suitably combining (1.3), (2.16), (3.6) and (3.7), we can also obtain (3.5).

We now apply the relationship (3.5) to derive some further consequences of Theorem 2.3. Indeed, in light of (3.5), the assertion (2.12) of Theorem 2.3 assumes the following form in the two-variable case.

COROLLARY 3.2. For every polynomial $\mathscr{P}_{m}(x)$ of degree $m$ in $x$,

$$
\begin{aligned}
\sum_{k=0}^{p} \mathscr{P}_{m}(k) g_{k}^{\left(-\alpha-n_{1}, \alpha+\beta+n_{1}+n_{2}+1-k\right)}(x, y) g_{p-k}^{(\alpha,-\alpha-\beta+1-p+k)}(x, y) \\
=\delta_{p, n_{1}+n_{2}}(y-x)^{n_{1}} y^{n_{2}} \mathscr{P}_{m}(\alpha+\beta+p)+\frac{(-1)^{m}}{m !} \mathscr{P}_{m}^{(m)}(0) \\
\cdot\left[\delta_{n_{1}, m-1}(\alpha)_{m} x^{n_{2}}(x-y)^{p-n_{2}}+(-1)^{p} \delta_{n_{2}, m-1}(\beta)_{m} x^{n_{1}} y^{p-n_{1}}\right] \\
\\
\left(m, p, n_{1}, n_{2} \in \mathbb{N}_{0} ; n_{1}, n_{2} \geq m-1 ; p \geq n_{1}+n_{2}\right) .
\end{aligned}
$$

REMARK 9. By setting $n_{1}=n_{2}=m$ in (3.8), we deduce the following summation identity for every polynomial $\mathscr{P}_{m}(x)$ of degree $m$ in $x$ :

$$
\begin{aligned}
& \sum_{k=0}^{p} \mathscr{P}_{m}(k) g_{k}^{(-\alpha-m, \alpha+\beta+2 m+1-k)}(x, y) g_{p-k}^{(\alpha,-\alpha-\beta+1-p+k)}(x, y) \\
& \quad=\delta_{p, 2 m}\left(y^{2}-x y\right)^{m} \mathscr{P}_{m}(\alpha+\beta+p) \quad\left(m, p \in \mathbb{N}_{0} ; p \geq 2 m\right)
\end{aligned}
$$

By successively applying the relationships (3.5), (1.3) and (3.6), we find that

$$
\begin{aligned}
g_{n}^{(\alpha, \beta-n)}(x, y) & =g_{n}^{(\alpha,-\alpha-\beta+1)}(x-y,-y)=(-x)^{n} P_{n}^{(-\alpha-n, \alpha+\beta-n-1)}\left(\frac{x-2 y}{x}\right) \\
& =x^{n} P_{n}^{(\alpha+\beta-n-1,-\alpha-n)}\left(\frac{2 y-x}{x}\right),
\end{aligned}
$$

which, upon setting $y=x(x+1) / 2$, would yield

$$
P_{n}^{(\alpha+\beta-n-1,-\alpha-n)}(x)=x^{-n} g_{n}^{(\alpha, \beta-n)}\left(x, \frac{x(x+1)}{2}\right) .
$$


REMARK 10. In light of the relationship (3.9), the assertion (3.8) of Corollary 3.2 can be used to derive the following consequence for every polynomial $\mathscr{P}_{m}(x)$ of degree $m$ in $x$ :

$$
\begin{aligned}
\sum_{k=0}^{p} \mathscr{P}_{m}(k) P_{k}^{\left(\beta+n_{2}-k, \alpha+n_{1}-k\right)}(x) P_{p-k}^{(-\beta-p+k,-\alpha-p+k)}(x) \\
=\delta_{p, n_{1}+n_{2}} 2^{-p}(x-1)^{n_{1}}(x+1)^{n_{2}} \mathscr{P}_{m}(\alpha+\beta+p)+\frac{(-1)^{m}}{m !} \mathscr{P}_{m}^{(m)}(0) \\
\cdot\left[\delta_{n_{1}, m-1}(\alpha)_{m}\left(\frac{1-x}{2}\right)^{p-n_{2}}+(-1)^{p} \delta_{n_{2}, m-1}(\beta)_{m}\left(\frac{1+x}{2}\right)^{p-n_{1}}\right] \\
\left(m, p \in \mathbb{N}_{0} ; n_{1}, n_{2} \geq m-1 ; p \geq n_{1}+n_{2}\right),
\end{aligned}
$$

which, upon setting $n_{1}=n_{2}=m$, leads us at once to (2.19).

If we define

$$
\varphi_{k}^{\left(\alpha_{2}, \cdots, \alpha_{r}\right)}(\mathbf{x}):=\lim _{\left|\alpha_{1}\right| \rightarrow \infty}\left\{g_{k}^{(\alpha)}\left(\frac{x_{1}}{\alpha_{1}}, x_{2}, \cdots, x_{r}\right)\right\},
$$

then it is easily observed from (1.4), (1.5) and (1.6) that

$$
\begin{aligned}
\varphi_{k}^{\left(\alpha_{2}, \cdots, \alpha_{r}\right)}(\mathbf{x}) & =\sum_{k_{1}+\cdots+k_{r}=k}\left(\alpha_{2}\right)_{k_{2}} \cdots\left(\alpha_{r}\right)_{k_{r}} \frac{x_{1}^{k_{1}}}{k_{1} !} \cdots \frac{x_{r}^{k_{r}}}{k_{r} !} \\
\sum_{k=0}^{\infty} \varphi_{k}^{\left(\alpha_{2}, \cdots, \alpha_{r}\right)}(\mathbf{x}) z^{k} & =e^{x_{1} z} \prod_{j=2}^{r}\left\{\left(1-x_{j} z\right)^{-\alpha_{j}}\right\} \quad\left(|z|<\min \left\{\left|x_{2}\right|^{-1}, \cdots,\left|x_{r}\right|^{-1}\right\}\right)
\end{aligned}
$$

and

$$
\begin{aligned}
& \sum_{k=0}^{\infty} k^{n} \varphi_{k}^{\left(\alpha_{2}, \cdots, \alpha_{r}\right)}(\mathbf{x}) z^{k} \\
& =e^{x_{1} z} \prod_{j=2}^{r}\left\{\left(1-x_{j} z\right)^{-\alpha_{j}}\right\} \sum_{k=0}^{n} k ! \cdot S(n, k) \cdot \varphi_{k}^{\left(\alpha_{2}, \cdots, \alpha_{r}\right)}\left(x_{1}, \frac{x_{2}}{1-x_{2} z}, \cdots, \frac{x_{r}}{1-x_{r} z}\right) z^{k} \\
& \quad\left(|z|<\min \left\{\left|x_{2}\right|^{-1}, \cdots,\left|x_{r}\right|^{-1}\right\} ; n \in \mathbb{N}_{0}\right) .
\end{aligned}
$$

We thus arrive at the following immediate consequence of Theorem 2.3.

THEOREM 3.3. For every polynomial $\mathscr{P}_{m}(x)$ of degree $m$ in $x$,

$$
\begin{gathered}
\sum_{k=0}^{p} \mathscr{P}_{m}(k) \varphi_{k}^{\left(-\alpha_{2}-n_{2}, \cdots,-\alpha_{r}-n_{r}\right)}\left(-x_{1}, x_{2}, \cdots, x_{r}\right) \varphi_{p-k}^{\left(\alpha_{2}, \cdots, \alpha_{r}\right)}(\mathbf{x}) \\
=\delta_{p, n_{2}+\cdots+n_{r}+m} \frac{(-1)^{p}}{m !} \mathscr{P}_{m}^{(m)}(0) x_{1}^{m} \prod_{j=2}^{r}\left\{x_{j}^{n_{j}}\right\}+\frac{(-1)^{m}}{m !} \mathscr{P}_{m}^{(m)}(0) \psi_{2}(\boldsymbol{\alpha}) \\
\left(m, p, n_{2}, \cdots, n_{r} \in \mathbf{N}_{0} ; n_{j} \geq m-1(j=2, \cdots, r) ; p \geq n_{2}+\cdots+n_{r}+m\right) .
\end{gathered}
$$


Finally, if we define

$$
\Phi_{k}^{\left(\alpha_{1}\right)}(\mathbf{x})=\lim _{\min \left\{\left|\alpha_{2}\right|, \cdots,\left|\alpha_{r}\right| \mid \rightarrow \infty\right.}\left\{g_{k}^{(\alpha)}\left(x_{1}, \frac{x_{2}}{\alpha_{2}}, \cdots, \frac{x_{r}}{\alpha_{r}}\right)\right\},
$$

then (1.4), (1.5) and (1.6) would readily yield the following properties:

$$
\begin{gathered}
\Phi_{k}^{\left(\alpha_{1}\right)}(\mathbf{x})=\sum_{k_{1}+\cdots+k_{r}=k}\left(\alpha_{1}\right)_{k_{1}} \frac{x_{1}^{k_{1}}}{k_{1} !} \cdots \frac{x_{r}^{k_{r}}}{k_{r} !} \\
\sum_{k=0}^{\infty} \Phi_{k}^{\left(\alpha_{1}\right)}(\mathbf{x}) z^{k}=\left(1-x_{1} z\right)^{-\alpha_{1}} e^{\left(x_{2}+\cdots+x_{r}\right) z} \quad\left(|z|<\left|x_{1}\right|^{-1}\right) \\
\sum_{k=0}^{\infty} k^{n} \Phi_{k}^{\left(\alpha_{1}\right)}(\mathbf{x}) z^{k} \\
=\left(1-x_{1} z\right)^{-\alpha_{1}} e^{\left(x_{2}+\cdots+x_{r}\right) z} \cdot \sum_{k=0}^{n} k ! \cdot S(n, k) \Phi_{k}^{\left(\alpha_{1}\right)}\left(\frac{x_{1}}{1-x_{1} z}, x_{2}, \cdots, x_{r}\right) z^{k} \\
\quad\left(|z|<\left|x_{1}\right|^{-1} ; n \in \mathbb{N}_{0}\right) .
\end{gathered}
$$

and

Thus, by making use of the assertion (2.12) of Theorem 2.3 once again, we can immediately deduce Theorem 3.4 below.

THEOREM 3.4. For every polynomial $\mathscr{P}_{m}(x)$ of degree $m$ in $x$,

$$
\begin{aligned}
\sum_{k=0}^{p} \mathscr{P}_{m}(k) \Phi_{k}^{(-\alpha-n)}\left(x_{1},-x_{2}, \cdots,-x_{r}\right) \Phi_{p-k}^{(\alpha)}(\mathbf{x}) & \begin{cases}\delta_{p, m+n} \frac{(-1)^{p}}{m !} \mathscr{P}_{m}^{(m)}(0) x_{1}^{n} x_{2}^{m}+\delta_{n, m-1} \frac{(-1)^{m}}{m !} \mathscr{P}_{m}^{(m)}(0)(\alpha)_{m} x_{1}^{p} & (r=2) \\
\delta_{n, m-1} \frac{(-1)^{m}}{m !} \mathscr{P}_{m}^{(m)}(0)(\alpha)_{m} x_{1}^{p} & (r \geq 3)\end{cases} \\
& \left(m, p, n \in \mathbb{N}_{0} ; n \geq m-1 ; p \geq m+n ; r \in \mathbb{N} \backslash\{1\}\right) .
\end{aligned}
$$

Numerous further corollaries and consequences of the results presented in this paper can be derived similarly.

\section{Acknowledgements}

The present investigation was supported, in part, by the National Science Council of the Republic of China under Grant NSC-95-2115-M-032-007 and, in part, by 
the Natural Sciences and Engineering Research Council of Canada under Grant OGP0007353.

\section{References}

[1] W.-C. C. Chan, C.-J. Chyan and H. M. Srivastava, "The Lagrange polynomials in several variables", Integral Transform. Spec. Funct. 12 (2001) 139-148.

[2] K.-Y. Chen and H. M. Srivastava, "A new result for hypergeometric polynomials", Proc. Amer. Math. Soc. 133 (2005) 3295-3302.

[3] A. Erdélyi, W. Magnus. F. Oberhettinger and F. G. Tricomi, Higher Transcendental Functions, Volume III (McGraw-Hill Book Company, New York, Toronto and London, 1955).

[4] A. Alın, E. Erkuş and F. Taşdelen, "The $q$-Lagrange polynomials in several variables", Taiwanese J. Math. 10 (2006) 1131-1137.

[5] E. Erkuş and A. Alın, "A note on the Lagrange polynomials in several variables", J. Math. Anal. Appl. 310 (2005) 139-148.

[6] E. Erkuş and H. M. Srivastava, "A unified presentation of some families of multivariable polynomials", Integral Transform. Spec. Funct. 17 (2006) 315-320.

[7] H. M. Srivastava and H. L. Manocha, A Treatise on Generating Functions (Halsted Press (Ellis Horwood Limited, Chichester), John Wiley and Sons, New York, Chichester, Brisbane and Toronto, 1984).

[8] G. Szegö, Orthogonal Polynomials, Volume 23 of American Mathematical Society Colloquium Publications, fourth edition (American Mathematical Society, Providence, Rhode Island, 1975).

[9] I. Tomescu, Problems in Combinatorics and Graph Theory, Wiley-Interscience Series in Discrete Mathematics, Translated from the Romanian by R. A. Melter (A Wiley-Interscience Publication, John Wiley and Sons, New York, Chichester, Brisbane and Toronto, 1985). 